\title{
Acceptance to Use Daily Oral Pre-Exposure Prophylaxis (PrEP) as an HIV Prevention Method and Ability to Pay for PrEP among Men Who Have Sex with Men in Ho Chi Minh City, Vietnam
}

\author{
Huong Thi Thu Phan ${ }^{1 *}$, Nga Thi Thu Vu ${ }^{2}$ \\ ${ }^{1}$ Vietnam Administration of HIV/AIDS Prevention and Control, Ministry of Health, Hanoi, Vietnam \\ ${ }^{2}$ Institute of Preventive Medicine and Public Health, Hanoi Medical University, Hanoi, Vietnam \\ Email: ^huongphanmoh@gmail.com,nga.vuthithu@gmail.com
}

How to cite this paper: Phan, H.T.T. and Vu, N.T.T. (2017) Acceptance to Use Daily Oral Pre-Exposure Prophylaxis (PrEP) as an HIV Prevention Method and Ability to Pay for PrEP among Men Who Have Sex with Men in Ho Chi Minh City, Vietnam. Health, 9, 1326-1336.

https://doi.org/10.4236/health.2017.99096

Received: May 31, 2017

Accepted: September 19, 2017

Published: September 22, 2017

Copyright $\odot 2017$ by authors and Scientific Research Publishing Inc. This work is licensed under the Creative Commons Attribution International License (CC BY 4.0).

http://creativecommons.org/licenses/by/4.0/

\begin{abstract}
Background: Men who have sex with men (MSM) are disproportionately affected by HIV in Vietnam. Regardless of international and national agencies' efforts, the HIV epidemic in MSM has been increasing in recent years. Novel and evidence-based HIV antiretroviral pre-exposure prophylaxis (PrEP) maybe needed to combat the HIV epidemic among this population in Vietnam. This study aims to identify how MSM accept the use of PrEP as an HIV prevention method and their ability to pay for it. Method: This is a cross-sectional study of 373 MSM seeking HIV testing in a community-based HIV clinic in Ho Chi Minh City, Vietnam from January to April 2016. Potential participants were briefly informed about ethical considerations, screened for eligibility, and signed an informed consent form when selected. Face-to-face interviews were conducted by the clinic's staff. Descriptive and analytical analysis was performed by STATA 13.0. Results: Of 373 participants, only 92 men (24.7\%) reported having known about PrEP. Out of 360 men who were questioned about their acceptance of PrEP as an HIV prevention method, 274 (76.1\%) embraced the use of PrEP. The median maximum affordable monthly PrEP cost was one million Vietnam Dong (VND) (equivalent to US\$43) and the median average affordable monthly PrEP cost was 0.5 million VND (approximately $\$ 21.7)$. The majority of men (68.8\%) could afford an average of less than $\$ 43$ a month for PrEP. Conclusion: There is a relatively high level of PrEP acceptance among MSM in Ho Chi Minh City, Vietnam. To increase PrEP acceptance, raising awareness and understanding of PrEP is recom-
\end{abstract}


mended. To expand PrEP interventions in Vietnam, the target population's ability to pay should be a key focus.

\section{Keywords}

Pre-Exposure Prophylaxis, Men Who Have Sex with Men, Vietnam, Acceptability to Use PrEP, Ability to Pay

\section{Introduction}

HIV was first reported in Vietnam in the early 1990s [1], and the epidemic has rapidly spread through the country during the last 30 years, reported in all provinces and cities since 1999 [2]. In its early stages, the epidemic was concentrated in several high-risk populations, such as people who inject drugs and female sex workers. People who inject drugs were the dominant route of transmission, accounting for over $88 \%$ of new cases during the late 1990s [2] [3]. As reported by the Vietnam Administration of HIV/AIDS Control Office (VAAC) at the end of 2015, the cumulative number of reported HIV cases throughout the country was 227,154 , of which 85,194 had progressed to AIDS and 86,176 had died of AIDS [4]. From the beginning of the epidemic until the mid-2000s, MSM were not identified as a priority population in Vietnam and were largely ignored in Vietnam's HIV surveillance and intervention efforts [5] [6].

Since the early 2000s, there have been warning signs of HIV spreading among MSM in Vietnam [7]. An early study of MSM in Ho Chi Minh City pointed out that MSM who engaged in a number of risky sexual behaviors, such as condomless anal intercourse (CAI) and having multiple sexual partners, had inaccurate knowledge about HIV prevention and transmission [8]. Nonetheless, it was only in the late 2000s that MSM were officially identified as a high-risk population for HIV infection [9]. Since the mid-2000s, there have been several reports and published papers from National HIV/AIDS Sentinel Surveillance, Integrated Biological and Behavioral Surveillance (IBBS), and community-based studies that assess the magnitude of HIV infection among MSM in Vietnam. While IBBS found an increasing HIV prevalence in MSM in major cities during the 2000s and early 2010s, there was a declining trend from 2013. Meanwhile, studies in smaller southern provinces found a lower prevalence [10] [11] [12] [13] [14]. Unfortunately, data from longitudinal cohort studies for estimation of HIV incidence are lacking.

Evidence from trials in the past few years has demonstrated that PrEP is effective in preventing new HIV infections among several high-risk populations, including MSM [15]. In a multinational trial study of PrEP (IPreX) [16], the combination of tenofovir and emtricitabine reduced the incidence of new HIV cases by $44 \%$. The combination of tenofovir and emtricitabine has been approved as a PrEP indication for HIV high-risk individuals by the US Food and Drug Ad- 
ministration (FDA) [15]. A recent systematic review and meta-analysis of the effectiveness of the tenofovir and emtricitabine combination or tenofovir alone showed an HIV incidence reduction of $49 \%$ and $33 \%$, respectively [17]. It has been demonstrated that PrEP uptake, adherence, and retention is well-tolerated, particularly among people self-perceived to have a high risk of HIV infection [18]. Recent studies also reported reasonable cost-effectiveness of PrEP in several HIV high-risk populations [19]. PrEP is recommended as a strategy to reduce HIV transmission among homosexual, bisexual, and other MSM by World Health Organization and public health managers [20] [21]. Nonetheless, the rollout of PrEP worldwide is slow and PrEP coverage is limited [22]. One of the barriers to PrEP expansion is awareness among MSM [15]. A recent meta-analysis of 68 published articles reported a low level of PrEP acceptability among MSM (57.8\%, 95\% Interval Confidence: 52.4\% - 63.1\%) [23]. An online study in 2015 in Vietnam showed $26.8 \%$ of participating MSM had heard about PrEP, and $68.8 \%$ of participants could only afford a monthly cost of less than $\$ 9$ for PrEP [24]. The study also indicated that $65.7 \%$ of men preferred rectal microbicide and approximately $34 \%$ preferred PrEP. However, the findings of this study may not be applicable to the wider MSM community in Vietnam since it recruited participants from online social networks.

Vietnam Ministry of Health is currently enthusiastic about piloting PrEP as an HIV prevention method for MSM in Vietnam. To implement a successful program, understanding of whether MSM are interested in PrEP, whether they are willing to use PrEP as an HIV prevention method, and whether PrEP is financially affordable are crucial considerations. However, an updated understanding of these factors in Vietnam is limited. This study aims to identify the acceptability or willingness to use PrEP as an HIV prevention method and the ability to pay for PrEP among MSM who access private, community-based HIV testing facilities in Ho Chi Minh City, Vietnam.

\section{Method}

This is a cross-sectional study conducted from January to April 2016 in a community-based HIV testing clinic in Ho Chi Minh City, Vietnam. A convenience sample was derived from MSM who came to this clinic for HIV testing. All MSM clients were verbally informed about ethical considerations of the study, screened for eligibility, and signed an informed consent inform if selected. Face-to-face interviews were conducted by experienced data-collectors belonging to the clinic's staff. In the interviews, all clients, including men who expressed having any prior knowledge about PrEP, were given a brief introduction to PrEP, its purpose, eligibility, and basic requirements while taking PrEP. Each interview lasted for approximately 25 to 30 minutes. To be deemed eligible, MSM had to be 18 years or older at the time of interview, have reported having anal sex with at least one man in the previous three months, and consent to participate in the study. The study received local ethical approval from Hanoi Uni- 
versity of Public Health, Vietnam.

\subsection{Data Collection}

The questionnaire was developed in Vietnamese, pre-tested, and revised as needed before actual interviews were conducted. Men were asked about:

- Socio-demographic characteristics:

o Age: men were asked about their date of birth. Age was calculated from subtracting this date from the study's fieldwork end date.

o Sexual orientation: men were asked if they were homosexual, bisexual, or had another sexual orientation.

o Education: men were asked for the highest level of their education. Men whose highest education was from secondary school or below were classified as having a low educational level. Men who finished high school or had some vocational training were classified as having a medium educational level. Men with a college education and above were classified as having a high educational level.

o Occupation: men were asked whether they were a student or employed with the government or a private/foreign company. Men were grouped together according to their job category, including a group for the unemployed.

o Income: men were asked about their total monthly income (in VND).

o Involvement in selling sex. men were asked if they ever sold sex by either receiving money or gifts from another man.

o HIV risk: men were asked about their self-perceived HIV infection risk using a 5-level Likert scale. Men who replied with having a low or very low HIV risk were classified as having a low self-perceived HIV risk. Men who answered with having a medium HIV risk were classified as having a medium self-perceived HIV risk. Men who responded with having a high or very high HIV risk were classified as having a high self-perceived HIV risk.

- HIV Pre-exposure prophylaxis (PrEP)

o Knowledge of PrEP. men were asked a Yes/No question whether they had any prior knowledge of PrEP.

o Acceptability to use PrEP: men were asked a Yes/No question whether they accept PrEP as an HIV prevention method. Men also had the option to not answer this question.

o The average acceptable monthly cost of PrEP. men were asked how much they could afford to pay for monthly use of PrEP (in VND).

o The maximum acceptable monthly cost of PrEP. men were asked for the highest monthly price they could afford to pay for use of PrEP.

o Desire for further PrEP information: men were asked a multiple-choice question about PrEP information they wished to acquire, including who should use PrEP, PrEP's effectiveness, requirements during treatment, side effects, disadvantages, and costs. If they wanted to know any other information about PrEP, they were asked to specify. 


\subsection{Data Analysis}

Electronic form for data entry was developed by Epidata 3.1. Descriptive and analytical statistics were performed by Stata version 13.0 (Stata Corp, College Station, TX, USA). We reported frequencies and percentages for demographical and outcome categorical variables and mean (standard deviation [SD]) or median (Inter quartile range [IQR]) for continuous variables. We performed a chi-square test to compare the distribution of the willingness to use PrEP by demographical and other personal variables.

\section{Results}

Participants' demographical characteristics are presented in Table 1. Of 373 participants, $85.5 \%$ self-reported as homosexual, $13.1 \%$ claimed to be bisexual, and only $1.3 \%$ reported having another sexual orientation. The participants' median age was 23.7 (IQR: 18.0 - 47.5), among which the majority were between 20 and 30 years old, $9.4 \%$ were under 20 years old, and $11.2 \%$ were over 40 years old. Over two third of participants had a college-level or higher education (73.7\%). $21.5 \%$ had finished high school or have had some vocational training. Only 4.8\% of participants had an education level lower than high school. With regards to

Table 1. Participants' demographical characteristics $(\mathrm{N}=373)$.

\begin{tabular}{|c|c|c|}
\hline Sexual orientation & $\mathbf{N}$ & $\%$ \\
\hline Homosexual & 319 & 85.5 \\
\hline Bisexual & 49 & 13.1 \\
\hline Other & 5 & 1.3 \\
\hline Age (medium, IQR) & \multicolumn{2}{|c|}{$23.7(18.0-47.5)$} \\
\hline$<20$ & 35 & 9.4 \\
\hline $20-30$ & 294 & 78.8 \\
\hline$>30$ & 44 & 11.2 \\
\hline \multicolumn{3}{|l|}{ Education } \\
\hline Less than high school & 18 & 4.8 \\
\hline High school \& vocational training & 80 & 21.5 \\
\hline College and above & 275 & 73.7 \\
\hline \multicolumn{3}{|l|}{ Occupation } \\
\hline Student & 124 & 33.2 \\
\hline Government staff & 23 & 6.2 \\
\hline Private company staff & 132 & 35.4 \\
\hline Foreign company staff & 33 & 8.9 \\
\hline Other & 61 & 16.4 \\
\hline Income (medium, IQR) (million VND) & \multicolumn{2}{|c|}{$5.0(0.6-24.0)$} \\
\hline Ever sold sex & 30 & 8.0 \\
\hline
\end{tabular}


occupation, approximately one third of participants identified as students (33.2\%), and yet another third worked for a private company (35.4\%). $6.2 \%$ of participants were government employees, $8.9 \%$ worked for a foreign company, and $16.4 \%$ worked in other job categories. The median monthly income was 5 million VND (equivalent to approximately \$217). Of 373 participants, 30 (8.0\%) self-reported any involvement in ever selling sex.

Of 373 participants, 92 men (24.7\%) reported having known about PrEP. Of the 360 men who answered the question asking about their willingness to use PrEP after hearing its explanation, 274 men (76.1\%) accepted use of PrEP as an HIV prevention method. The distributions of willingness to use PrEP by demographical variables are presented in Table 2.

Bisexual and other sexual orientations had a higher acceptance or willingness to use PrEP compared to homosexual men (80.8\% versus $75.3 \%)$. The highest

Table 2. Distributions of willingness to use PrEP as an HIV prevention method by participants' demographical characteristics $(\mathrm{N}=360)$.

\begin{tabular}{|c|c|c|c|c|}
\hline & \multicolumn{2}{|c|}{ Willing to use PrEP } & \multirow[t]{2}{*}{$\mathrm{X}^{2}$} & \multirow[t]{2}{*}{$P$ value } \\
\hline & Yes & No & & \\
\hline Sexual orientation & & & & $0.483^{*}$ \\
\hline Homosexual & $232(75.3)$ & $76(24.7)$ & & \\
\hline Bisexual and other & $42(80.8)$ & $10(19.2)$ & & \\
\hline Age (medium, IQR) & & & 1.705 & 0.426 \\
\hline$<20$ & $24(68.6)$ & $11(31.4)$ & & \\
\hline $20-30$ & $218(77.6)$ & $63(22.4)$ & & \\
\hline$>30$ & $32(72.7)$ & $12(27.3)$ & & \\
\hline Education & & & 0.04 & 0.98 \\
\hline Less than high school & $13(76.5)$ & $4(23.3)$ & & \\
\hline High school \& vocational training & $60(76.9)$ & $18(23.1)$ & & \\
\hline College and above & $201(75.9)$ & $64(24.2)$ & & \\
\hline Occupation & & & 3.539 & 0.472 \\
\hline Student & $89(76.1)$ & $28(23.9)$ & & \\
\hline Government staff & $15(65.2)$ & $8(34.8)$ & & \\
\hline Private company staff & $104(79.4)$ & $27(20.6)$ & & \\
\hline Foreign company staff & $21(67.7)$ & $10(32.3)$ & & \\
\hline Other & $45(77.6)$ & $13(22.4)$ & & \\
\hline Self-perceived HIV risk & & & 0.420 & 0.81 \\
\hline Low & $151(75.5)$ & $49(24.5)$ & & \\
\hline Medium & $71(74.7)$ & $24(25.3)$ & & \\
\hline High & $49(79.0)$ & $13(20.1)$ & & \\
\hline Know about PrEP & & & & $0.044^{*}$ \\
\hline No & $199(73.4)$ & $72(26.6)$ & & \\
\hline Yes & $75(84.3)$ & $14(15.7)$ & & \\
\hline
\end{tabular}

*: Fisher exact. 
percentages of men willing to use PrEP are men who are aged between 20 and 30 years old $(77.6 \%)$, are staff of a private company $(79.4 \%)$, area student $(76.1 \%)$, or belong to other job categories (77.6\%). Men who had a high self-perceived HIV risk are more likely to be willing to use PrEP (79.0\%) compared to men with a medium risk $(74.7 \%)$ or with a low risk (75.5\%). However, all of these differences were not statistically significant. There is no significant difference of willingness to use PrEP by education distribution either.

Participants' ability to pay is presented in Table 3. The median maximum affordable monthly PrEP cost is 1 million VND (equivalent to \$43) (IQR: 0.1 5.0). The median average affordable monthly PrEP cost is 0.5 million VND (equivalent to \$21.7) (IQR: 0.05 - 4.0). The largest percentage of men could afford monthly PrEP when costs were between 0.5 and 1 million VND (44.4\%), followed by when the cost was below 0.5 million VND (24.4\%). Approximately $21 \%$ of men could pay for PrEP when costs ranged from 1 million to 1.5 million VND. Only $10.6 \%$ of participants could afford a monthly cost of over 1.5 million VND.

Participants' desire to know more about PrEP is demonstrated in Table 4. The majority of participants wanted to know about the effectiveness of PrEP (69.5\%) and side effects of PrEP (59.7\%). Additional information they wished to know about PrEP was the cost of PrEP (52.0\%) and the requirements while taking PrEP (42.8\%). A small proportion of participants wanted to know about disadvantages of PrEP (37.3\%) and who should use PrEP (36.8\%).

Table 3. Ability to pay for PrEP (million Vietnam Dong*).

\begin{tabular}{ccc}
\hline & $\mathrm{n}$ & $\%$ \\
\hline Median maximum affordable monthly PrEP cost & $1.0(0.1-5.0)$ & \\
Median reasonable monthly PrEP cost & $0.5(0.05-4.0)$ & \\
Level of PrEP affordability & 90 & 24.4 \\
$<0.5$ & 164 & 44.4 \\
$\geq 0.5-<1.0$ & 76 & 20.6 \\
$\geq 1.0-<1.5$ & 39 & 10.6 \\
\hline
\end{tabular}

*: Vietnam's currency. One USD is equivalent to approximately 23,000 VND.

Table 4. Participants' desire to know about $\operatorname{PrEP}(\mathrm{N}=367)$.

\begin{tabular}{ccc}
\hline Who should use PrEP & $\mathrm{n}$ & $\%$ \\
Effectiveness of PrEP & 135 & 36.8 \\
Requirement while taking PrEP & 255 & 69.5 \\
Side effects of PrEP & 157 & 42.8 \\
Disadvantages of PrEP & 219 & 39.7 \\
Cost of PrEP & 137 & 37.3 \\
\hline
\end{tabular}




\section{Discussion}

Our study found several useful suggestions for HIV prevention interventions for MSM in Vietnam. With regards to the implementation of PrEP, we found a relatively high acceptance or willingness to use PrEP among participating MSM in Ho Chi Minh City, Vietnam. Willingness to use PrEP is 76.1\%, higher than the previously reported pooled estimate of PrEP acceptability (57.8\%) from a previous meta-analysis [23]. A review of studies that report PrEP acceptability among MSM indicated a wide range of 33\% - 80\% among HIV-negative MSM [25]. This review reported high acceptability of PrEP in a number of countries, including Australia, US, Canada, Peru, Thailand, India, and China. Our findings are also higher than the reported acceptability to use PrEP (approximately 34\%) among MSM from a previous study in Vietnam [24].

From our study, men more likely to accept PrEP either are between 20 and30 years old, work for a private company, area student, belong to other job categories, or have a high self-perceived risk of HIV infection. However, these differences are not statistically significant. Studies in other settings indicated that PrEP acceptability is higher among older men and men with a higher self-perceived HIV risk [25]. The high acceptability rate suggests PrEP is accepted by men with various socio-demographic backgrounds, and implementation in MSM in Vietnam has potential.

Our findings indicate that prior knowledge of PrEP is associated with a higher willingness to use PrEP. In our study, $24.7 \%$ of participants reported having prior knowledge of PrEP. This finding runs parallel with findings in previous studies in Vietnam [24] and North America [25]. Together, our findings suggest that dissemination of PrEP information would increase the acceptability of using PrEP as an HIV prevention method among MSM in Vietnam. Our participants were particularly interested in hearing more information on the efficacy and side effects of PrEP. They were also very interested in the associated costs and the requirements while taking $\operatorname{PrEP}$.

One of the challenges in implementing and expanding PrEP, particularly in middle and low-income countries, is its relatively high cost [15] [25]. A previous study in Vietnam reported that the majority of their participating MSM could only afford a monthly PrEP payment of less than $\$ 9$. Our study showed that half of the participants could afford to pay a maximum monthly PrEP cost of 1 million VND (approximately $\$ 43$ ), the majority of men could afford a reasonable monthly payment of 0.5 million VND (\$21.7), and only one fifth could afford a monthly payment of less than 0.5 million VND (equivalent to \$21.7). Future pilots and implementation of PrEP in Vietnam should consider these levels of affordability. To lower the cost of PrEP, medications that are slow release or have long-lasting effects should be considered [15]. Additionally, to implement PrEP for MSM in Vietnam, public health managers should also pay attention to other challenges of PrEP rollout, including maintenance of optimal adherence to PrEP, fear of long-term side effects from both service providers and beneficiaries [15], 
and fear of PrEP-associated stigma [18].

Several considerations should be kept in mind while interpreting our study's findings. Due to the cross-sectional nature and the convenience sample derived from an HIV-testing clinic's clients in Ho Chi Minh City, our study's findings may not be generalized to the broader MSM population in this city. Our assessment of acceptability for PrEP was based on single questions, such as, "Do you accept use of PrEP as an HIV prevention method for yourself" and a three-category answer, "Yes", "No", and "No answer". The decision to take PrEP may depend on a number of factors [25]. Therefore, this assessment may not reflect the true level of PrEP acceptability among our participants.

\section{Conclusion}

We found a relatively high level of PrEP acceptance among MSM in Ho Chi Minh City, Vietnam. To increase PrEP acceptance, raising awareness and understanding about PrEP is recommended. To expand PrEP interventions in Vietnam, interventions should pay attention to people's ability to pay. To reduce PrEP cost, generic, slow-release, or other long-lasting PrEP medications are recommended.

\section{Acknowledgements}

We acknowledge the great support and input from staff of GLINK in Ho Chi Minh City, Vietnam during the data collection process. We thank PATH for their technical assistance in PrEP introduction and comments on the manuscript.

\section{Conflict of Interest}

There is no conflict of interest declared.

\section{Contributions}

HTTP led the development of the research protocol and data collection tools, undertook data collection and data analysis, wrote the initial draft of the manuscript, and prepared the final manuscript. NV and VTTN provided input on the research protocol and data collection and contributed to the manuscript.

\section{References}

[1] Nguyen, T.H. and Wolffers, I. (1994) HIV Infection in Vietnam. Lancet, 343, 410. https://doi.org/10.1016/S0140-6736(94)91239-4

[2] Quan, V.M., Chung, A., Long, H.T. and Dondero, T.J. (2000) HIV in Vietnam: The Evolving Epidemic and the Prevention Response, 1996 through 1999. Journal of Acquired Immune Deficiency Syndromes, 25, 360-369. https://doi.org/10.1097/00126334-200012010-00011

[3] Nguyen, T.H., Nguyen, T.L. and Trinh, Q.H. (2004) HIV/AIDS Epidemics in Vietnam: Evolution and Responses. AIDS Education and Prevention: Official Publication of the International Society for AIDS Education, 16, 137-154. 
https://doi.org/10.1521/aeap.16.3.5.137.35527

[4] Viet Nam Administration of HIV/AIDS Control Office (VAAC) (2016) HIV/AIDS Prevention and Control Report for 2015 Hanoi, Vietnam 2016.

http://vaac.gov.vn/Cms Data/Contents/Vaac/Folders/Solieubaocao/Solieu/ conten ts/BCG2DGP6NQ77KBCX/Bao-cao-2015 so-lieu_Final.pdf.

[5] Blanc, M.-E. (2005) Social Construction of Male Homosexualities in Vietnam. Some Keys to Understanding Discrimination and Implications for HIV Prevention Strategy. International Social Science Journal, 57, 661-673. https://doi.org/10.1111/j.1468-2451.2005.583.x

[6] The Social Republic of Vietnam (2006) HIV/AIDS Second Country Report on Following up to the Declaration of Commitment on HIV/AIDS. Declaration of Commitment on HIV/AIDS Adopted at the 26th United Nations General Assembly Special Session in June 2001 (UNGASS). The Social Republic of Vietnam, Hanoi.

[7] Colby, D., Cao, N.H. and Doussantousse, S. (2004) Men Who Have Sex with Men and HIV in Vietnam: A Review. AIDS Education and Prevention: Official Publication of the International Society for AIDS Education, 16, 45-54. https://doi.org/10.1521/aeap.16.1.45.27722

[8] Colby, D.J. (2003) HIV Knowledge and Risk Factors among Men Who Have Sex with Men in Ho Chi Minh City, Vietnam. Journal of Acquired Immune Deficiency Syndromes, 32, 80-85.https://doi.org/10.1097/00126334-200301010-00012

[9] The Socialist Republic of Vietnam (2009) Declaration of Commitment on HIV and AIDS Adopted at the 26th United Nations General Assembly Special Session in June 2001 (UNGASS). The Socialist Republic of Vietnam, Hanoi.

[10] Vietnam Ministry of Health (2011) Results from the HIV/STI Biological and Behavioral Surveillance (IBBS) in Vietnam-Round II 2009. Vietnam Ministry of Health, Hanoi.

[11] Vietnam Ministry of Health (2006) Results from the HIV/STI Integrated Biological and Behavioural Surveillance (IBBS) in Vietnam, 2005-2006. Hanoi, Vietnam.

[12] National Institute of Hygiene and Epidemiology (Vietnam Ministry of Health) (2014) HIV/STI Integrated Biological and Behavioural Surveillance (IBBS) in Vietnam. Results from Round III and Trends across Three Round (2005-2009-2013) of Survey. Hanoi, Vietnam.

[13] Pham, Q.D., Nguyen, T.V., Hoang, C.Q., Cao, V., Khuu, N.V., Phan, H.T., et al. (2012) Prevalence of HIV/STIs and Associated Factors among Men Who Have Sex with Men in An Giang, Vietnam. Sexually Transmitted Diseases, 39, 799-806. https://doi.org/10.1097/OLQ.0b013e318265b180

[14] Nguyen, T.V., Van Khuu, N., Nguyen, P.D., Tran, H.P., Phan, H.T., Phan, L.T., et al. (2016) Sociodemographic Factors, Sexual Behaviors, and Alcohol and Recreational Drug Use Associated with HIV among Men Who Have Sex with Men in Southern Vietnam. AIDS and Behavior, 20, 2357-2371.

https://doi.org/10.1007/s10461-015-1265-x

[15] Krakower, D.S. and Mayer, K.H. (2015) Pre-Exposure Prophylaxis to Prevent HIV Infection: Current Status, Future Opportunities and Challenges. Drugs, 75, 243-251. https://doi.org/10.1007/s40265-015-0355-4

[16] Grant, R.M., Lama, J.R., Anderson, P.L., McMahan, V., Liu, A.Y., Vargas, L., et al. (2010) Pre-Exposure Chemoprophylaxis for HIV Prevention in Men Who Have Sex with Men. New England Journal of Medicine, 363, 2587-2599. https://doi.org/10.1056/NEJMoa1011205

[17] Okwundu, C.I., Uthman, O.A. and Okoromah, C.A. (2012) Antiretroviral Pre-Exposure Prophylaxis (PrEP) for Preventing HIV in High-Risk Individuals. The 
Cochrane Database of Systematic Reviews, No. 7, Cd007189. https://doi.org/10.1002/14651858.CD007189.pub3

[18] Mugo, N.R., Ngure, K., Kiragu, M., Irungu, E. and Kilonzo, N. (2016) The Pre-Exposure Prophylaxis Revolution; from Clinical Trials to Programmatic Implementation. Current Opinion in HIV and AIDS, 11, 80-86. https://doi.org/10.1097/COH.0000000000000224

[19] Cambiano, V., Miners, A. and Phillips, A. (2016) What Do We Know about the Cost-Effectiveness of HIV Pre-Exposure Prophylaxis, and Is It Affordable? Current Opinion in HIV and AIDS, 11, 56-66. https://doi.org/10.1097/COH.0000000000000217

[20] Beyrer, C. (2014) Strategies to Manage the HIV Epidemic in Gay, Bisexual, and Other Men Who Have Sex with Men. Current Opinion in Infectious Diseases, 27, 1-8. https://doi.org/10.1097/QCO.0000000000000037

[21] World Health Organization (WHO) (2015) Guideline on When to Start Antiretroviral Therapy and on Pre-Exposure Prophylaxis for HIV. World Health Organization, Geneva.

[22] Beyrer, C., Baral, S.D., Collins, C., Richardson, E.T., Sullivan, P.S., Sanchez, J., et al. (2016) The Global Response to HIV in Men Who Have Sex with Men. The Lancet (London, England), 388, 198-206.

[23] Peng, P., Su, S., Fairley, C.K., Chu, M., Jiang, S., Zhuang, X., et al. (2017) A Global Estimate of the Acceptability of Pre-Exposure Prophylaxis for HIV among Men Who Have Sex with Men: A Systematic Review and Meta-Analysis. AIDS and Behavior. https://doi.org/10.1007/s10461-017-1675-Z

[24] Oldenburg, C.E., Le, B., Huyen, H.T., Thien, D.D., Quan, N.H., Biello, K.B., et al. (2016) Antiretroviral Pre-Exposure Prophylaxis Preferences among Men Who Have Sex with Men in Vietnam: Results from a Nationwide Cross-Sectional Survey. Sexual Health. https://doi.org/10.1071/SH15144

[25] Holt, M. (2014) HIV Pre-Exposure Prophylaxis and Treatment as Prevention: A Review of Awareness and Acceptability among Men Who Have Sex with Men in the Asia-Pacific Region and the Americas. Sexual Health, 11, 166-170.

\section{Submit or recommend next manuscript to SCIRP and we will provide best} service for you:

Accepting pre-submission inquiries through Email, Facebook, LinkedIn, Twitter, etc. A wide selection of journals (inclusive of 9 subjects, more than 200 journals)

Providing 24-hour high-quality service

User-friendly online submission system

Fair and swift peer-review system

Efficient typesetting and proofreading procedure

Display of the result of downloads and visits, as well as the number of cited articles

Maximum dissemination of your research work

Submit your manuscript at: http://papersubmission.scirp.org/

Or contact health@scirp.org 